\title{
Adherencia de la suplementación con multimicronutrientes y nivel de hemoglobina en niños de 6 a 36 meses del distrito de Huanca Huanca, Perú, 2017
}

\author{
Adherence of multi-micronutrient supplementation and hemoglobin level in children \\ from 6 to 36 months of Huanca Huanca district, Peru, 2017
}

\author{
Andrea Tinoco Ramos ${ }^{1}$, Kerlly Yanzapanta Cruz ${ }^{2}$, Keila Miranda Limachi ${ }^{3}$
}

\begin{abstract}
RESUMEN
Objetivo del estudio fue determinar los factores de adherencia a la suplementación con multimicronutrientes relacionados al nivel de hemoglobina en niños de 6 a 36 meses de edad del distrito de Huanca Huanca, Huancavelica. Material y métodos: el estudio fue de enfoque cuantitativo, de diseño no experimental, tipo correlacional y de corte transversal. La muestra fue no probabilística de tipo intencional, utilizando los criterios de inclusión y exclusión, conformada por 60 niños de 6 a 36 meses de edad. El instrumento utilizado para la recolección de datos fue el Test de Adherencia a la Suplementación con Multimicronutrientes de Espichan (2013) y la medición de hemoglobina se realizó con el Hemocue 201. Resultados: El 53,3\% de madres presentó una adherencia media. EI $53,3 \%$ de niños presentó hemoglobina normal. Además, no existe relación entre la adherencia general a la suplementación con multimicronutrientes y el nivel de hemoglobina, con un p-valor de 0,385 y se encontró una correlación baja en el factor relacionado al suplemento con un p-valor de 0,042. Conclusión: Ambas variables no se relacionan.
\end{abstract}

Palabras claves: Anemia, adherencia, factores, hemoglobina, multimicronutrientes.

\begin{abstract}
Objective: of the study was to determine the factors of adherence to supplementation with multimicronutrients related to the level of hemoglobin in children from 6 to 36 months of age in the district of Huanca Huanca, Huancavelica. Material and methods: the study was quantitative, nonexperimental, correlational and transversal design approach. The sample was not probabilistic of intentional type, using the inclusion and exclusion criteria, and consisted of 60 children from 6 to 36 months of age. The instrument used for the data collection was the adhesion test for multimicronutrient supplementation from Espichan (2013) and the hemoglobin measurement was performed with Hemocue 201. Results found that $53.3 \%$ of the mothers had an average adherence. $53.3 \%$ of the children had normal hemoglobin. In addition, there is no relationship between the general adherence to multimicronutrient supplementation and the hemoglobin level, with a p-value of 0.385 and a low correlation was found in the factor related to the supplement with a p-value of 0.042 . Conclussion: that both variables are not related.
\end{abstract}

Keywords: Anemia, adherence, factors, hemoglobin, multi-micronutrient. 


\section{INTRODUCCIÓN}

La anemia es una patología en la que el número de glóbulos rojos y, por ende, su trabajo de mayor importancia que es el de transportar oxígeno a todo el organismo, es insuficiente para satisfacer los requerimientos del mismo. Los requerimientos orgánicos específicos sufren variaciones dependiendo del género, la edad y la altitud sobre el nivel del mar en la que habita con mayor frecuencia el individuo, así mismo la práctica de hábitos nocivos y los diversos ciclos de la gestación (Organización Mundial de la Salud, 2011).

Según la Organización Mundial de la Salud (2008) la anemia afecta a nivel mundial a 1620 millones de personas, lo que corresponde al $24,8 \%$ de la población. Asimismo, la mayor prevalencia se da en los niños en edad preescolar $(47,4 \%)$, y la menor en los varones (12,7\%). Sin embargo, el grupo de población que cuenta con el mayor número de personas con nivel de hemoglobina por debajo de los valores normales, es el de las mujeres no embarazadas (468 millones). Por otro lado, a nivel de Latinoamérica y el Caribe, el Banco Mundial estima que 7,2 millones de niños menores de 5 años tienen un retraso del crecimiento, y 22,5 millones padecen de anemia. Así también, la mayor prevalencia de malnutrición crónica y de anemia se presenta durante la edad crítica de entre 6 y 24 meses. También el Banco Mundial en su estudio "Cómo proteger y promover la nutrición de las madres y los niños" recalcan que "Intervenir en la nutrición durante los primeros meses de vida tiene mucho sentido desde el punto de vista económico" (Soteras, 2012).

La realidad en el Perú es similar. Según, Román et al. (2014) la anemia en los niños menores de 5 años presenta una alta prevalencia en el Perú, pese a que en los últimos 20 años se han implementado algunos avances respecto a su prevención y tratamiento. Según un estudio realizado en la población a nivel nacional y regional denominado como la Encuesta Nacional Demográfica de Salud (ENDES), que utiliza una muestra probabilística, por capas y en diversas etapas, en el año 2013 encontraron que a nivel nacional, uno de cada tres niños menores de cinco años padecen de anemia (34\%), siendo un problema mayor en niños que residen en áreas rurales $(39,5 \%)$ en comparación a los que residen en áreas urbanas $(31,1 \%)$.
Frente a esta problemática, se han elaborado estrategias para poder contrarrestar el porcentaje de niños que padecen esta patología, la terapéutica más conocida es la administración de sulfato ferroso a dosis terapéuticas (3-6 mg/ $\mathrm{kg} / \mathrm{día}$ ) y evaluar la respuesta eritropoyética. La positividad de la prueba puede establecerse por un pico reticulocitario de los 5 a 10 días 0 un aumento de hemoglobina $\geq 1 \mathrm{~g} / \mathrm{dl}$ a los 30 días (Comité Nacional de Hematología, 2009). Como estrategia adjunta en algunos países se ha implementado la administración de Multimicronutrientes como lo menciona la Directiva Sanitaria, que establece la Suplementación Preventiva con Hierro en las niñas y niños menores de tres años, como parte de esta estrategia también se establece el seguimiento y monitoreo con el fin de evaluar la aceptación y adherencia al suplemento de hierro que esté recibiendo el menor (Ministerio de Salud, 2016). En consecuencia, a la necesidad de seguimiento y monitoreo se han realizado diversos estudios con respecto a la adherencia al consumo de multimicronutrientes.

La anemia en niños menores de 3 años es un ámbito de preocupación mundial, ya que padecerla en esta etapa de vida tiene consecuencias a largo plazo. Entre ellas principalmente está el desempeño cognitivo deficiente, lo que repercutirá en la adquisición de las capacidades en sus primeros años de vida, esto se verá evidenciado en logros educativos deficientes y en la adquisición de enfermedades infecciosas y el aumento de morbilidad $y$ mortalidad. A pesar de las estrategias aplicadas por el Ministerio de Salud, los estudios han demostrado que existe poca adherencia a la administración de suplementos de hierro. Según lo observado por las investigadoras en el distrito de Huanca Huanca, del total de niños menores de 3 años, el $80 \%$ padece de anemia a pesar de haber recibido el tratamiento, presentando los síntomas característicos de la anemia, evidenciando así la poca adherencia al consumo de suplementos de hierro y a una dieta rica en nutrientes, por todo lo expuesto, planteamos el siguiente objetivo. Determinar los factores de adherencia a la suplementación con multimicronutrientes relacionados al nivel de hemoglobina en niños de 6 a 36 meses de edad del distrito de Huanca Huanca, Huancavelica.

El estudio, será de beneficio para los niños en la prevención de la anemia, que ocasiona daños al nivel psicomotor y cognoscitivo, además permitirá 
sensibilizar acerca de la anemia y la adherencia a la suplementación con multimicronutrientes en las madres cuidadoras y las repercusiones que conlleva su alta prevalencia, de esta manera, las familias tendrán una mejor calidad de vida y aportarán para el desarrollo sostenible de su comunidad y región.

\section{MATERIALES Y MÉTODOS}

Esta investigación es de enfoque cuantitativo, de diseño no experimental, de corte transversal, y de tipo correlacional.

La población estuvo representada por 79 niños; la muestra estuvo conformada por 60 niños de 6 a 36 meses pertenecientes a los Puestos de Salud Ccarapa y Huanca Huanca. La selección de la muestra se obtuvo mediante el muestreo no probabilístico y de carácter censal, utilizando los criterios de inclusión y exclusión, para determinar la cantidad de participantes.

Se utilizó el Test de adherencia a la suplementación de Espichán (2013) con multimicronutrientes elaborada por. Este test tiene el objetivo de identificar los factores de adherencia a la suplementación en niños de 6 a 36 meses.

Para la recolección de datos del estudio, se realizó un convenio con el Puesto de Salud y el Municipio de Huanca Huanca, a los cuales se solicitó la autorización respectiva mediante un documento facilitado por la Escuela Profesional de Enfermería de la Universidad Peruana Unión. Posteriormente, se ejecutóla recolección de datos con la explicación respectiva del consentimiento informado. La aplicación del cuestionario se realizó durante los meses de junio a noviembre a través de visitas domiciliarias, respetando el horario previsto por cada participante y con una duración de 45 minutos.

\section{RESULTADOS}

En la tabla 1 se observa que del $100 \%$ de niños que reciben la suplementación, el 53,3\% presentó una adherencia media, seguido por el $46,7 \%$ con una adherencia alta; y no se encontró adherencia baja.

Tabla 1

Adherencia general a la suplementación con multimicronutrientes en niños de 6 a 36 meses de edad del distrito de Huanca Huanca, Huancavelica, 2017.

\begin{tabular}{|c|c|c|}
\hline Adherencia & n & $\%$ \\
\hline Alta & 28 & 46,7 \\
\hline Media & 32 & 53,3 \\
\hline Baja & 0 & 0 \\
\hline Total & 60 & 100 \\
\hline
\end{tabular}

En la tabla 2 se observa que del $100 \%$ de niños que recibieron la suplementación con multimicronutrientes, el $68,3 \%$ presentó una adherencia media con respeto al factor social, el $21,7 \%$ alta y el $10 \%$ baja. Así mismo, en el factor relacionado al personal de salud se encontró que, el 78,3\% evidenció una adherencia media y el $21,7 \%$ alta. Seguidamente, en el factor relacionado a la enfermedad, el 53,3\% manifestó una adherencia media, el $28,3 \%$ baja y el $18,3 \%$ alta. Por otro lado, en el factor relacionado al suplemento, el $50 \%$ mostró una adherencia alta al igual que la media, y no se encontró adherencia baja. Finalmente, en el factor relacionado a la persona que suministra el tratamiento, el $65 \%$ demostró una adherencia alta, el 35\% media y no se halló adherencia baja. 
Adherencia de la suplementación con multimicronutrientes y nivel de hemoglobina en niños de 6 a 36 meses del distrito de Huanca Huanca, Perú, 2017

Tabla 2

Factores de adherencia a la suplementación con multimicronutrientes en niños de 6 a 36 meses de edad del distrito de Huanca Huanca, Huancavelica, 2017.

\begin{tabular}{lcccccccc} 
Factores relacionados a la & \multicolumn{2}{c}{ Alto } & \multicolumn{3}{c}{ Medio } & \multicolumn{2}{c}{ Bajo } & \multicolumn{2}{c}{ Total } \\
\cline { 2 - 10 } Adherencia & $n$ & $\%$ & $n$ & $\%$ & $n$ & $\%$ & $n$ & $\%$ \\
\hline Social & 13 & 21,7 & 41 & 68,3 & 6 & 10 & 60 & 100 \\
Relacionado al personal de salud & 13 & 21,7 & 47 & 78,3 & 0 & 0 & 60 & 100 \\
Relacionado a la enfermedad & 11 & 18,3 & 32 & 53,3 & 17 & 28,3 & 60 & 100 \\
Relacionado al suplemento & 30 & 50 & 30 & 50 & 0 & 0 & 60 & 100 \\
Relacionado a la persona que & 39 & 65 & 21 & 35 & 0 & 0 & 60 & 100 \\
suministra el tratamiento & & & & & & & & \\
\hline
\end{tabular}

En la tabla 3 se observa que del 100\% de niños con suplementación, el 53,3\% presentó hemoglobina dentro de los parámetros normales, seguido por el $28,3 \%$ que evidenció anemia leve y el 18,3\% anemia moderada.

Tabla 3

Nivel de Hemoglobina en niños de 6 a 36 meses de edad del distrito de Huanca Huanca, Huancavelica, 2017.

\begin{tabular}{ccc} 
Hemoglobina & $\mathrm{n}$ & $\%$ \\
Normal $(11-14 \mathrm{~g} / \mathrm{dl})$ & 32 & 53,3 \\
Anemia leve $(10-10.9 \mathrm{~g} / \mathrm{dl})$ & 17 & 28,3 \\
Anemia moderada $(7-9.9 \mathrm{~g} / \mathrm{dl})$ & 11 & 18,3 \\
Anemia severa $(<7 \mathrm{~g} / \mathrm{dl})$ & 0 & 0 \\
Total & 60 & 100 \\
\hline
\end{tabular}

En la tabla 4 se encontró que del $100 \%$ de niños que recibieron suplementación con multimicronutrientes, el $28,3 \%$ indicó una adherencia alta con un nivel de hemoglobina normal, asimismo, el 18,3\% con adherencia media manifestó una anemia leve. De manera contradictoria, un $8,3 \%$ de niños con alta adherencia presentó anemia moderada. Se se obtuvo un p-valor de 0,385 y un coeficiente de correlación de $-0,107$, lo que indica que no existe relación entre la adherencia a la suplementación con multimicronutrientes y el nivel de $\mathrm{Hb}$ de los niños de 6 a 36 meses de edad.

Tabla 4

Relación entre la adherencia general a la suplementación con multimicronutrientes y nivel de hemoglobina en niños de 6 a 36 meses de edad del distrito de Huanca Huanca, Huancavelica, 2017.

\begin{tabular}{|c|c|c|c|c|c|}
\hline \multirow{2}{*}{$\begin{array}{c}\text { Adherencia } \\
\text { General }\end{array}$} & \multicolumn{5}{|c|}{ Nivel de Hemoglobina } \\
\hline & & Normal & Anemia leve & Anemia moderada & Total \\
\hline \multirow{2}{*}{ Adherencia Alta } & $\mathrm{n}$ & 17 & 6 & 5 & 28 \\
\hline & $\%$ & 28,3 & 10 & 8,3 & 46,7 \\
\hline \multirow{2}{*}{ Adherencia Media } & $\mathrm{n}$ & 15 & 11 & 6 & 32 \\
\hline & $\%$ & 25 & 18,3 & 10 & 53,3 \\
\hline \multirow{2}{*}{ Adherencia Baja } & $\mathrm{n}$ & 0 & 0 & 0 & 0 \\
\hline & $\%$ & 0 & 0 & 0 & 0 \\
\hline \multirow{2}{*}{ Total } & $\mathrm{n}$ & 32 & 17 & 11 & 60 \\
\hline & $\%$ & 53,3 & 28,3 & 18,3 & 100 \\
\hline
\end{tabular}

Tau-b = -, 107; $p=, 385$ 
En la tabla 5 se observa que según la prueba de correlación, el factor relacionado al suplemento fue el único que indicó una correlación negativa baja.

Tabla 5

Factores relacionados a la adherencia y nivel de hemoglobina en los niños de 6 a 36 meses de edad del distrito de Huanca Huanca, Huancavelica, 2017.

\begin{tabular}{lcc}
\multicolumn{1}{c}{ Factores de Adherencia } & Tau-b & p \\
Factor Social & $-0,152$ & 0,239 \\
Factor relacionado al personal de salud & $-0,043$ & 0,739 \\
Factor relacionado a la enfermedad & $-0,075$ & 0,491 \\
Factor relacionado al suplemento & $-0,241$ & 0,042 \\
Factor relacionado a la persona que suministra & $-0,149$ & 0,228 \\
el tratamiento & & \\
\hline
\end{tabular}

\section{DISCUSIÓN}

La Organización Mundial de la Salud (OMS) conjuntamente con la Fundación para Niños de las Naciones Unidas (UNICEF) calculan que la cantidad afectada de niños con anemia ferropénica es superior a los 750 millones, principalmente en los países en desarrollo, estableciéndose como una de las principales causas de los elevados porcentajes de morbimortalidad infantil (Urquidi, Mejía, y Vera, 2009). En el Perú, la anemia forma parte de un grave problema de salud pública con una prevalencia del $43,6 \%$ entre los niños de 6 a 35 meses y del $59,3 \%$ en niños de 6 a 12 meses (Ministerio de Salud, 2017). En el área urbana afecta al $31,1 \%$ mientras que en el área rural al $39,8 \%$. El Instituto Nacional de Salud menciona que, el departamento de Huancavelica en el año 2013 , reportó niños afectados con anemia en un $40,5 \%$, sobre todo con edades entre 6 y 59 meses (Román et al., 2015). Por otro lado, Munares y Gómez (2016), en su estudio para considerar la adherencia multimicronutrientes y factores asociados en sitios centinela, concluyeron que hubo una baja prevalencia de adherencia para un punto de corte exigente.

En el trabajo se encontró que el nivel de adherencia de los cuidadores de niños menores de 36 meses fue en un 53,3\% media, $46,7 \%$ alta y $0 \%$ baja (ver tabla 7). Resultados similares reportaron Juarez y Madueño (2016) en el estudio "Factores socioculturales y su relación con la adherencia al suplemento de multimicronutrientes en madres que acuden al Centro de Salud Santa Fe, Callao", donde encontraron que el $60 \%$ de los participantes presentó un nivel de adherencia adecuado y el 40\% inadecuado. También Espichán (2013), en el estudio "Factores de adherencia a la suplementación con sprinkles asociados al incremento de hemoglobina en niños de 6 a 60 meses, de asentamientos humanos del Distrito de San Martín de Porres", indicó que el $64 \%$ de niños(as) tuvo una adherencia alta al tratamiento. En contraposición, Hinostroza (2015) en la investigación "Barreras y motivaciones en el nivel de adherencia a la suplementación con multimicronutrientes en madres de niños menores de 36 meses, Cercado de Lima", señaló que solo el $8,5 \%$ de las madres tuvo una alta adherencia y el $91,5 \%$, baja.

La Organización Mundial de la Salud (2003) define a la adherencia como el grado en que la conducta de un paciente, en relación con la toma de medicación, el seguimiento de una dieta o la modificación de hábitos de vida, corresponde con las recomendaciones acordadas con el profesional sanitario. Del mismo modo, el Ministerio de Salud (2017a) menciona que la adherencia incluye la buena disposición para seguir el tratamiento en las dosis, horario y tiempo indicado; asimismo, se considera que es adecuada cuando se consume el $75 \%$ a más de la dosis indicada. Por tanto, Matsui (2007) recomienda incluir estrategias conductuales y educativas que permitan mejorar la adherencia, a pesar de no existir un consenso en la elección de estas. A su vez, invoca la necesidad de incluir a los niños y padres en la planificación del tratamiento, tomando en cuenta las barreras que puedan existir, tales como la frecuencia y el momento del consumo del medicamento y adaptarlos al estilo de vida y rutina diaria del niño y la familia. Cabe resaltar, que entre los principales beneficios de la suplementación con 
multimicronutrientes (MMN) está el incremento de la hemoglobina, que según Munayco et al. (2013) en un estudio realizado en regiones andinas del Perú, logró disminuir la prevalencia de anemia en niños de un $70,2 \%$ a un $36,6 \%$.

Según Morán (2012), uno de los principales factores que disminuyen la adherencia al tratamiento con sales de hierro son los variados efectos colaterales que presentan, como: las náuseas, vómitos, diarreas y el estreñimiento (Rodríguez, 2016). Sin embargo, Mayca, Medina, Velásquez, y Llanos (2017), indican que el rechazo al consumo de estos MMN está basado en representaciones sociales, como las creencias y costumbres, y que en su gran mayoría evidencian la desconfianza y poca aceptabilidad de la población al producto. A su vez, esto se ve influenciado por la sensibilización recibida, la cual no solamente debe ser verbal, sino también de contacto visual (evidencias palpables), y deben reforzarse en cada consulta para que la madre confíe más en el producto.

En cuanto al nivel de hemoglobina, se encontró que el $53 \%$ de los niños indicaron una hemoglobina normal y el $46,6 \%$ entre leve y moderada (ver tabla 9). De igual manera, Quispe y Mendoza (2016) en la investigación "Micronutrientes y su relación con la anemia en niños menores de 36 meses de edad del centro de salud Ciudad Blanca 2016", reportó que el $85 \%$ de los niños que consumieron los micronutrientes no presentaron anemia mientras que el $15 \%$ manifestaron una anemia leve. Por otro lado, Huamán et al. (2012) en su estudio "Consumo de suplementos con multimicronutrientes chispitas y anemia en niños de 6 a 35 meses: estudio transversal en el contexto de una intervención poblacional en Apurímac, Perú" hallaron que la prevalencia de anemia fue de $51,3 \%$. En contraste, Becerril (2011) en la investigación "Eficacia del sulfato ferroso y multimicronutrientes en el incremento de hemoglobina en los niños de 6 a 36 meses de edad, en los distritos de San Juan de Rontoy y Llamellin, provincia Antonio Raimondi, Ancash" encontró que el $66,7 \%$ de los niños presentaron algún grado de anemia. Además, numerosos estudios demuestran que las consecuencias de la anemia por deficiencia de hierro pueden deteriorar el desarrollo cognitivo, motor $y$ conductual (Ip, Hyder, Haseen, Rahman, y Zlotkin, 2009). Seguidamente, se encontró que no existe relación entre los factores de adherencia a la suplementación con multimicronutrientes y el nivel de hemoglobina con un p-valor de 0,385 (ver tabla 10). De igual manera Espichán (2013) en su estudio realizado en Perú, recalca que no existe relación significativa entre ambas variables. En torno a esto, Ocaña (2014) señala que sin el uso correcto de los micronutrientes y una orientación adecuada a los padres de familia sobre la importancia, preparación y administración de las Chis Paz será imposible prevenir y aportar de forma efectiva la reversión de la patología. Por otro lado, Buitrago (2011) manifiesta que el incumplimiento o falta de adherencia constituye un problema complejo que engloba una gran variedad de situaciones y presenta múltiples causas, del cual es preciso conocer su prevalencia real para cada entidad o enfermedad con la finalidad de combatirlo y mejorar el nivel de salud del paciente.

Datos descriptivos encontrados en la investigación, explican también la no relación hallada entre las variables, ya que del $28,3 \%$ de los cuidadores con una adherencia alta al tratamiento, sus hijos presentaron un nivel de hemoglobina normal, asimismo, ningún cuidador evidencio una adherencia baja y niños con anemia moderada. Al revisar las características sociodemográficas de la población en estudio se pudo observar que el $47,4 \%$ de los cuidadores manifestó un nivel educativo de primaria incompleta (ver tabla 4). Según De Tejada, González, Márquez, y Bastardo (2005) en su estudio realizado en Venezuela sobre la escolaridad materna y desnutrición del hijo o hija, la educación y la salud se encuentran íntimamente vinculadas, ya que encontraron que a medida que el nivel de escolaridad materna disminuye es más probable que el niño o niña sufra de desnutrición.

Continuando con el factor relacionado al suplemento y el nivel de hemoglobina, se encontró que existe una correlación negativa con un p-valor de 0,042 y un coeficiente de correlación de -0,241 (ver tabla 14). Del mismo modo (Cutipa \& Salomé, 2016) encontraron que el factor relacionado al suplemento se encuentra asociado al incremento de hemoglobina en niños de 6 a 36 meses en el Centro de Salud Chupaca-2015, con un porcentaje de adherencia media, el valor estadístico es mayor al valor chi cuadrado tabulado. En contraposición, Espichán (2013) obtuvo en su estudio que no existe relación entre las variables con un p-valor de 0,442 . Numerosos estudios demuestran la efectividad de los multimicronutrientes en la 
reducción de la anemia y la aceptabilidad en el organismo de los niños; se demostró que el $50 \%$ al $90 \%$ de niños anémicos que recibieron el suplemento presentaron niveles de hemoglobina normales. Además, concluyeron que las madres consideraban que el suplemento es fácil de usar, de mezclar con los alimentos y es apropiado para la introducción de la alimentación complementaria, solo informaron la presencia de oscurecimiento de heces como efecto secundario (Zlotkin et al., 2005). Por otro lado, Lago (2015) menciona que las características del tratamiento que modulan la adherencia son: la dosificación (monodosis dan mejores resultados), la vía de administración (los niveles más altos se dan en tratamiento que requiere medicación directa como inyectables), niveles elevados de supervisión y registro, duración (las tasas de adherencia disminuyen con el tiempo), efectos secundarios indeseados favorecen el incumplimiento, sobre todo cuando el paciente comienza a sentirse mejor.

\section{Declaración de financiamiento y de conflictos de interés:}

El estudio fue financiado por los autores, quienes declaran no tener conflictos de interés.

\section{Correspondencia}

Andrea Tinoco Ramos

Correo electrónico: andreatr@upeu.edu.pe

Kerlly Yanzapanta Cruz

Correo electrónico: kerlly@upeu.edu.pe

\section{REFERENCIAS BIBLIOGRÁFICAS}

Becerril, N. (2011). Eficacia del sulfato ferroso y multimicronutrientes en el incremento de hemoglobina en los niños de 6 a 36 meses de edad, en los distritos de San Juan de Rontoy y Llamellin, provincia Antonio Raimondi, Ancash, 2011. Universidad Peruana Unión. Retrieved from http://repositorio.upeu.edu.pe/bitstream/ handle/UPEU/625/Neisi_Tesis_bachiller_2011. pdf?sequence=1\&isAllowed $=y$

Buitrago, F. (2011). Adherencia terapéutica. ¡Qué difícil es cumplir! Atención Primaria, 43(7), 343-344. https://doi.org/10.1016/J.APRIM.2011.05.002
Comité Nacional de Hematología. (2009). Anemia ferropénica. Guía de diagnóstico y tratamiento. Archivos Argentinos de Pediatría, 107(4), 353361.

Cutipa, B., \& Salomé, N. (2016). Factores de adherencia a la suplementación con Nutromix asociados al incremento de hemoglobina en niños de 6 a 36 meses, en el centro de la salud Chupaca-2015. Universidad Privada de Huancayo Franklin Roosevelt. Retrieved from http://repositorio.uroosevelt.edu.pe/xmlui/ bitstream/handle/ROOSEVELT/11/AVANCE FORMATEO TESIS ENFER \%28BERTA $Y$ NADIA\%29.pdf?sequence=1\&isAllowed=y

De Tejada, M., González, A., Márquez, Y., \& Bastardo, L. (2005). Escolaridad materna y desnutrición del hijo o hija. Centro Clínico Nutricional Menca de Leoni. Caracas. Anales Venezolanos de Nutrición, 18(2), 162-168. Retrieved from http://www.scielo.org.ve/scielo.php?script=sci_ arttext\&pid=S0798-07522005000200004

Espichán, P. (2013). Factores de adherencia a la suplementación con sprinkles asociados al incremento de hemoglobina en niños de 6 a 60 meses, de asentamientos humanos del Distrito de San Martín de Porres. Universidad Nacional de San Marcos.

Hinostroza, M. (2015). Barreras y motivaciones en el nivel de adherencia a la suplementación con multimicronutrientes en madres de niños menores de 36 meses, Cercado de Lima. Universidad Nacional Mayor de San Marcos. Retrieved from http://cybertesis.unmsm.edu.pe/bitstream/ cybertesis/4226/1/Hinostroza_fm.pdf

Huamán, L., Aparco, J., Nuñez, E., Gozáles, E., Pillaca, J., \& Mayta, P. (2012). Consumo de suplementos con multimicronutrientes chispitas y anemia en niños de 6 a 35 meses: estudio transversal en el contexto de una intervención poblacional en Apurímac, Perú. Revista Peruana de Medicina Experimental y Salud Publica, 29(3), 314-323. Retrieved from http://www.scielo.org. pe/scielo.php?script=sci_arttext\&pid=S172646342012000300004\&lng=es\&nrm=iso\&tIng=es

Ip, H., Hyder, S. M. Z., Haseen, F., Rahman, M., \& Zlotkin, S. H. (2009). Improved adherence and anaemia cure rates with flexible administration of micronutrient Sprinkles: a new public health approach to anaemia control. European Journal of Clinical Nutrition, 63(2), 165-172. https://doi. org/10.1038/sj.ejcn.1602917

Juarez, D., \& Madueño, A. (2016). Factores socioculturales y su relación con la adherencia al suplemento de multimicronutrientes en madres de acuden al Centro de Salud Santa Fe, Callao 2016. Universidad Nacional del Callao. 
Lago, N. (2015). Adherencia al tratamiento. Tendencias En Medicina, 46, 99-109. Retrieved from http://www.tendenciasenmedicina.com/ Imagenes/imagenes46/art_13.pdf

Matsui, D. (2007). Current Issues in Pediatric Medication Adherence. Pediatric Drugs, 9(5), 283-288. https://doi.org/10.2165/00148581200709050-00001

Mayca, J., Medina, A., Velásquez, J., \& Llanos, L. (2017). Representaciones sociales relacionadas a la anemia en niños menores de tres años en comunidades Awajún y Wampis, Perú. Revista Peruana de Medicina Experimental En Salud Pública, 34(3), 414-422. https://doi.org/10.17843/ rpmesp.2017.343.2870

Ministerio de Salud. (2016). Directiva sanitaria para la Prevención de Anemia mediante la Suplementación con Micronutrientes y Hierro en niñas y niños menores de 36 meses.

Ministerio de Salud. (2017). Norma Técnica de Salud para el manejo terapéutico y preventivo de la anemia en niños, adolescentes, mujeres gestantes y puérperas.

Munares, O., \& Gómez, G. (2016). Adherencia a multimicronutrientes y factores asociados en niños de 6 a 35 meses de sitios centinela, Ministerio de Salud, Perú. Revista Brasileira de Epidemiologia, 19(3), 539-553. https://doi. org/10.1590/1980-5497201600030006

Munayco, C., Ulloa, M., Medina, J., Lozano, C., Tejada, V., Castro, C., ... Arias, L. (2013). Evaluación del impacto de los multimicronutrientes en polvo sobre la anemia infantil en tres regiones Andinas del Perú. Revista Peruana de Medicina Experimental Y Salud Publica, 30(2), 229-234.

Ocaña, D. (2014). Impacto del programa de suplementación con micronutrientes para evitar anemia en niños de 6 meses a 2 años de edad en el subcentro de salud Picaihua, período enero-junio 2013. Universidad Técnica de Ambato. Retrieved from http://repositorio.uta. edu.ec/bitstream/123456789/8391/1/Ocaña Anzules\%2C Delia Cristina.pdf

Organización Mundial de la Salud. (2008). Worldwide prevalence of anaemia 1993-2005: WHO global database on anaemia.

Organización Mundial de la Salud. (2011). Concentraciones de hemoglobina para diagnosticar la anemia y evaluar su gravedad. Ginebra: Organización Mundial de la Salud.
Quispe, C., \& Mendoza, S. (2016). Micronutrientes y su relación con la anemia en niños menores de 36 meses de edad del Centro de Salud Ciudad Blanca 2016. Universidad Ciencias de la Salud Arequipa.

Rodríguez, J. (2016). Factores asociados con la adherencia en 3 meses a la Suplementación con Multimicronutrientes en niños entre 6 y 24 meses de edad, Chimbote, 2016.

Román, Y., Rodríguez, Y., Gutierrez, E., Aparco, J. P., Gómez, I., \& Fiestas, F. (2015). Anemia en la población infantil del Perú: aspectos clave para su afronte. Lima. Retrieved from http://www. ins.gob.pe/repositorioaps/0/4/jer/evidencias/ ANEMIA FINAL_v.03mayo2015.pdf

Soteras, A. (2012, December 7). Unos 22,5 millones de niños sufren anemia en Latinoamérica. Panamá. Retrieved from http://www.efesalud. com/unos-225-millones-de-ninos-sufren-anemiaen-latinoamerica/

Urquidi, C., Mejía, H., \& Vera, C. (2009). Adherencia al tratamiento de la anemia con fumarato ferroso microencapsulado. Archivos de Pediatría Del Uruguay, 80(2), 137-143. Retrieved from http:// www.scielo.edu.uy/pdf/adp/v80n2/v80n2a12.pdf

Zlotkin, S. H., Schauer, C., Christofides, A., Sharieff, W., Tondeur, M. C., \& Hyder, S. M. Z. (2005). Micronutrient Sprinkles to Control Childhood Anaemia. PLoS Medicine, 2(1), e1. https://doi. org/10.1371/journal.pmed.0020001

Recibido: $21 / 02 / 18$ Aceptado: 25/06/18 